Apidologie, 1975, 6 (2), 91-94.

\title{
DÉTERMINATION DU SULFATHIAZOLE DANS LES MIELS PAR CHROMATOGRAPHIE EN COUCHE MINCE
}

\author{
Sulfathiazol-Bestimmung im Honig mittels \\ der Dünnschicht-Chromatographie \\ Augusto GRANDI \\ Istituto di Zoocolture della Università - Perugia (Italie).

\section{SUMMARY} \\ DETERMINATION OF SULPHATIAZOL \\ IN HONEYS BY THIN-LAYER CHROMATOGRAPHY
}

A method is proposed for the determination of sulphatiazol in honeys.

$20 \mathrm{~g}$ of honey are extracted three times with $30 \mathrm{ml}$ of acetone at each time. Concentrated extracts are transferred into a chromatographic column which contains basic alumina (activity Brockmann III). The elution is earried out with $30 \mathrm{ml}$ of chloroform, then $5 \mathrm{ml}$ of acetone acidified by $1,5 \mathrm{ml}$ of $\mathrm{HCl} 2 \mathrm{~N}$, and further with $60 \mathrm{ml}$ of acetone. The last elution is collected, concentrated and dissolved in $0,5 \mathrm{ml}$ of acetone. The chromatography is achieved on a thin-layer of silica gel $H$ Merck using chloroform/methanol $(4 / 1)$ as developping solvent.

When the migration is over, the chromatogramm is air-dried before being sprayed with a reagent for visualization.

The sensitivity of the method is $0,1 \mathrm{ppm}$.

\section{RÉSUMÉ}

On propose une méthode pour la détermination du sulfathiazole dans les miels.

On extrait $20 \mathrm{~g}$ de miel par trois fois avec $30 \mathrm{ml}$ d'acétone chaque fois; les extraits sont concentrés dans une colonne chromatographique contenant de l'oxyde d'aluminium basique (activité Brockman III). On élue par $30 \mathrm{ml}$ de chloroforme, $5 \mathrm{ml}$ d'acétone acidifiés par 1,5 ml de $\mathrm{HCl} 2 \mathrm{~N}$ et par $60 \mathrm{ml}$ d'acétone. Le dernier éluat est recueilli, concentré et repris avec $0,5 \mathrm{ml}$ d'acétone. On effectue ensuite la chromatographie sur plaque de gel de silice H Merck, en employant comme solvant mobile le chloroforme/méthanol (4/1). 
Lorsque la migration est terminée, on fait sécher la plaque en plein air puis on vaporise le mélange révélateur.

La sensibilité de la méthode est de $0,1 \mathrm{ppm}$.

\section{INTRODUCTION}

Parmi les maladies des abeilles, la loque américaine est sans doute celle qui cause les préoccupations les plus grandes. En effet, en plus d'être extrêmement contagieuse, elle provoque, dans les colonies atteintes, des dégâts très importants qui vont jusqu'à la mort, si l'on n'intervient pas à temps.

Il s'agit d'une maladie du couvain à caractère épizootique, causée par Bacillus larvae WhIтE, pour laquelle les règlements sanitaires prévoient deux possibilités : la destruction des colonies infectées et les traitements chimiothérapiques.

On ne recourt au premier moyen que dans les cas extrêmes; dans les autres cas on procède à un traitement médicamenteux.

Les médicaments les plus utilisés dans la lutte contre Bacillus larvae sont essentiellement les sulfamides et les antibiotiques; parmi ceux-ci, le sulfathiazole et la terramycine se sont montrés les plus efficaces. Le sulfamide est préférable à l'antibiotique, car il est plus stable dans les conditions ambiantes de la ruche et il peut garder ultérieurement son efficacité dans le miel.

Actuellement on emploie le sulfathiazole sodique, car il a le mérite d'être entièrement soluble dans l'eau.

Les sulfamides, lorsqu'ils sont administrés au début du printemps et en automne aux concentrations conseillées $(0,5 \mathrm{~g}$ pour 4 litres de sirop de sucre), sont inoffensifs pour les abeilles et ne contaminent pas le miel. Mais si l'on exagère dans l'emploi et dans les doses, le produit passe dans le miel en portant préjudice à son caractère naturel.

Étant donné qu'il est possible d'effectuer des contrôles sur les échantillons pour en déterminer le taux de contamination, on a mis au point la méthode ci-dessous décrite.

\section{MATÉRIEL UTILISÉ ET TECHNIQUES EXPÉRIMENTALES}

\section{Réactifs et appareillages}

a) Standard : sulfathiazole ou sulfathiazole sodique dans l'acétone, à la concentration de $0,1 \mathrm{mg} / \mathrm{ml}$.

b) Gel de silice $H$ Merck, pour chromatographie en couche mince.

c) Solvant mobile : chloroforme/méthanol (4/1).

d) Détecteur : $1 \mathrm{~g}$ de p-Diméthylaminobenzaldéhyde dissout dans $100 \mathrm{ml}$ d'éthanol et additionné de $1 \mathrm{ml}$ d'HCl concentré.

e) Oxyde d'aluminium basique C. ERBA. 
f) Appareillage pour couches minces : plaque de verre $20 \times 5 \mathrm{~cm}$ avec une épaisseur de $0,25 \mathrm{~mm}$ de gel de silice $H$ Merck en suspension dans l'eau dans le rapport $1: 2,8$.

Les plaques sont prêtes à l'usage après les avoir laissé sécher en plein air.

\section{Méthode analytique}

On additionne $20 \mathrm{~g}$ de miel, placés dans un tube à centrifugation de $100 \mathrm{ml}$, avec $30 \mathrm{ml}$ d'acétone. On agite pendant 2 minutes environ; on centrifuge le mélange pendant 5 minutes à $2500 \mathrm{t} / \mathrm{min}$. On répète l'extraction encore deux fois.

On concentre à petit volume $(5 \mathrm{ml}$ environ) les extraits réunis. On les transfère ensuite avec $15 \mathrm{ml}$ d'acétone/DMF $5 / 1$ employés pour les lavages dans une colonne à chromatographie en verre d'un diamètre intérieur de $10 \mathrm{~mm}$. contenant $10 \mathrm{~g}$ d'oxyde d'aluminium hasique (activité Brockman III).

Ensuite, on élue par $30 \mathrm{ml}$ de chloroforme, $5 \mathrm{ml}$ d'acétone acidifiés par $1,5 \mathrm{ml}$ de $\mathrm{HCl} 2 \mathrm{~N}$ et enfin par $60 \mathrm{ml}$ d'acétone. Cet éluat, contrairement aux autres, est recueilli, porté à sec et repris avec $0,5 \mathrm{ml}$ d'acétone. La solution est placée sur la plaque chromatographique à des doses comprises entre $1 \mu \mathrm{l}$ et $10 \mu \mathrm{l}$ selon le taux de sulfamides contenus dans le miel. Après avoir séché les taches, la plaque est placée dans une cuve cylindrique, précédemment saturée par un mélange de chloroforme/méthanol (4/1).

Lorsque la séparation chromatographique est terminée, on fait sécher la plaque en plein air et ensuite on projette le mélange révélateur.

\section{DISCUSSION ET CONCLUSION}

La méthode proposée permet d'extraire du miel aussi bien le sulfathiazole que le sulfathiazole sodique, mais la séparation des deux substances n'est pas réalisable par chromatographie.

Dans la plaque traitée par le mélange révélateur, en plus de la tache jaune caractéristique, on relève deux autres taches, l'une jaune et l'autre violette, localisées respectivement au-dessous ( $3 \mathrm{~cm}$ environ) et au-dessus ( $1,5 \mathrm{~cm}$ environ) de celle de sulfathiazole. On attribue leur formation aux produits chimiques utilisés pour l'analyse, comme on a pu l'établir en faisant une épreuve à blanc.

Pour deux raisons on n'a pas jugé bon de poursuivre les recherches sur leur origine $: 1^{0} /$ on ne disposait pas de réactifs produits par d'autres maisons, $2^{\circ} /$ leur présence n'altérait pas du tout les résultats des analyses.

La méthode décrite se montre très utile, car elle permet de repérer d'une manière simple et sans possibilité d'interférences des quantités très petites de sulfathiazole (sensibilité $0,1 \mathrm{ppm}$.).

Req̧u pour publication en décembre 1974
Eingegangen im December 1974

\section{ZUSAMMENFASSUNG}

Es wird eine Methode zur Bestimmung von Sulfathiazol im Honig vorgelegt.

Methode : Dreimal $20 \mathrm{~g}$ Honig werden jeweils mit $30 \mathrm{ml}$ Azeton extrahiert. Die auf ein Volumen von etwa $5 \mathrm{ml}$ konzentrierten Auszüge werden mit $15 \mathrm{ml}$ Azeton (5:1), die für das Auswaschen benutzt wurden, in eine Chromatographensäule von $10 \mathrm{~mm}$ Innendurchmesser, 
die $10 \mathrm{~g}$ basisches Aluminiumoxyd (Aktivität Brockman III) enthält, gebracht. Dann wird mit $30 \mathrm{ml}$ Chloroform, einer Lösung von $5 \mathrm{ml}$ saurem Azeton, $1,5 \mathrm{ml} \mathrm{HCl} 2 \mathrm{~N}$ und $60 \mathrm{ml}$ Azeton eluiert. Das letzte Eluat wird, im Gegensatz zu den übrigen, aufgenommen, getrocknet und mit 1,5 ml Azeton erneut aufgenommen.

Die Lösung wird in unterschiedlichen Mengen (zwischen $1 \mu l$ und $10 \mu l$ ) auf eine $20 \times 5 \mathbf{c m}$ grosse Glasplatte, die $25 \mathrm{~mm}$ dick mit Silicium Gel Merck, suspendiert in $\mathrm{H}_{2} \mathrm{O}(1: 2,8)$, beschichtet wurde, aufgetragen und danach an der Luft getrocknet.

Die aufsteigende Chromatographie wird in einer zylindrischen Wanne durchgeführt, die zuvor mit Chloroform-Methanol $(4: 1)$ gesättigt wurde.

Wenn die Entwicklung beendet ist, lässt man die Platte an der Luft trocknen. Danach wird sie besprüht. Das Sprühmittel besteht aus $1 \mathrm{~g}$ p-Dimethylaminobenzaldehyd, das in $100 \mathrm{ml}$ Aethanol unter Zugabe von $1 \mathrm{ml}$ konzentrierter $\mathrm{HCl}$ gelöst wurde.

Ergebnisse : Die vorgeschlagene Methode erlaubt es, aus Honig sowohl Sulfathiazol als auch Natriumsulfathiazol zu extrahieren, die jedoch chromatographisch nicht getrennt werden. Ausser den Sulfathiazolflecken entdeckt man noch - über und unter diesen - zwei andere Flecken, die aber die Resultate nicht beeinträchtigen und deren Vorhandensein auf die benutzten Chemikalien zurückgeführt wird.

Der Wert der vorgelegten Methode wird zudem durch ihre Empfindlichkeit, die 0,1 ppm beträgt, bestätigt.

\section{RÉFÉRENCES BIBLIOGRAPHIQUES}

Begliomini A., Fravolini A., 1971. - Identificazione per cromatografia su strato sottile di sulfamidici nei mangimi. Archivio Veterinario Italiano, 22 (5), 273-278.

Crerr U.R., 1969. - Thin-Layer Chromatography and Ultraviolet Spectrophotometry of Sulfonamide Mixtures. A study of the absolute recoveries. J. Chromatogr., 4.5, 421-431.

Leland R.A., Stanley E. R., 1965. - Qualitative Determination of Sulfa Drugs in Medicated Feeds by Thin-Layer Chromatography. J. Assoc. Offic. Agr. Chemist., 48, 278-279.

Szalkowski C. R., 1973. - Determination of Sulfaquinoxaline in Feeds : Collaborative Study. J. Assoc. Offic. Agr. Chemists., 56, 758-761. 